\title{
Toward Laser Pulse Waveform Analysis for Scene Interpretation
}

\author{
Nicolas Vandapel, Omead Amidi and J. Ryan Miller \\ Carnegie Mellon University \\ The Robotics Institute \\ 5000 Forbes Avenue \\ Pittsburgh, PA 15213
}

\begin{abstract}
Laser based sensing for scene interpretation and obstacle detection is challenged by partially viewed targets, wiry structures, and porous objects. We propose to address such problems by looking at the laser pulse waveform. We designed a new laser sensor with off-the-shelf components. In this paper we report on the design and the evaluation of this low cost and compact sensor, suitable for mobile robot application. We determine classical parameters such as operation range, repeatability, accuracy, resolution, but we also analyze laser pulse waveforms modes and mode shape in order to extract additional information on the scene.
\end{abstract}

\section{INTRODUCTION}

Partially viewed targets, wiry structures and porous objects are still a challenge for laser based sensing and scene interpretation. Thin profile obstacles such as wires and objects concealed in vegetation are difficult to detect by laser range finder. Wire detection is particularly important for helicopters during Nap-Of-the-Earth flight (NOE). During low altitude above the canopy flight, power lines constitute a major danger that can bring down helicopters. Landing Medevac helicopters encounter this type of danger everyday. Ground mobile robots evolving in natural environments are also challenged by concertina wires, thin branches and hidden obstacles in vegetation. More, cluttered scenes are known to produce occlusions that produce to mixed-pixels in laser range measurements.

Our objective is to address such problems by looking at the laser pulses waveform. We integrate, in space and time, the sampled energy returned by targets after being illuminated by a pulsed laser to infer the structure of the scene perceived. By accumulating partial and uncertain information in a probabilistic framework, as is [1], we expect to be able to detect low signature objects. In addition we link the geometry of the target to the shape of laser pulse waveform. To demonstrate our approach, we conceived and implemented a new short range sensor capable of recording the complete laser pulse waveform. In this paper we report on the first phase of our work: the design of our prototype sensor and the evaluation of its performances.

Matthies [2] presented results on single point statistics computed from data from a single point laser range finder, to differentiate between vegetation and solid surface like rocks. In [3], he presents a more geometric approach to this problem. Another approach is to learn local 3-D points statistics and to perform Bayesian classification, as proposed in [4], [5]. The problem of mixed pixels detection received attention as in [6] or [7]. They rely on some continuous assumption about the geometry of the scene. Several systems exist for power line detection, based on Millimeter wave radar [8], laser range finder (HELLAS [9]), electromagnetic field sensor and IF camera (PODS [10]). Most of them are for military aircrafts, and they are designed to detect clusters of relatively large cables with no clutter.

In the next section, we review multi-echo lasers and pulse waveform analysis. We then describe our sensor, present results on the analysis of collected waveforms, report the performance of our sensor, and finally conclude.

\section{BEYOND SINGLE ECHO PER PULSE LASER}

\section{A. Sensors}

Almost all time-of-flight laser sensors commonly used in mobile robot applications measure the distance of, at most, a single echo per pulse emitted. This is true for Sick LMS291, Riegl LD90, or GDRS mobility ladar. They return either the first pulse or the last pulse. The exception is the new series of LMS-Z210/360/440 lasers by Riegl [11], which record both the first and last pulse. This high resolution/density laser has been used on mobile robots to perform static mapping of the environment [12], a complete scan taking 2:30 minutes. The limitations of such sensors with thin objects, occlusions and non-solid surface, is well known.

Multi-echo lasers are another class of sensors and are widely used by the remote sensing community to produce a digital terrain model of the Earth. More than 2 echos can be retrieved per pulse, assuming favorable target visibility conditions. The same community has access to laser that can record the complete laser pulse waveform. It is used to estimate biomass parameters or the canopy structure of forested terrains. See [13] for a review of the current airborne sensors. In addition of having a large foot-print, such sensors are too expensive and bulky to be used on-board mobile robots.

Flash ladar - focal array plane range finder - can also capture the complete waveform. But here the sensor projects a homogeneous plane of light instead of a focused beam [14]. Long range versions are still under development while short range version $(<7 \mathrm{~m}$ range) are commercially available, like the Zmini [15]. 


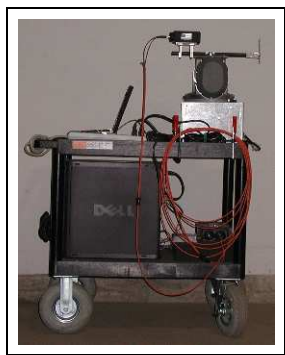

(a) Side view

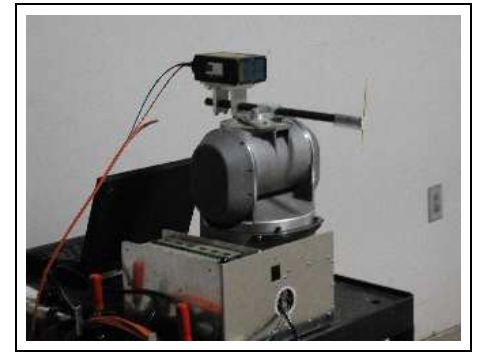

(b) Optical head, reference target and turret

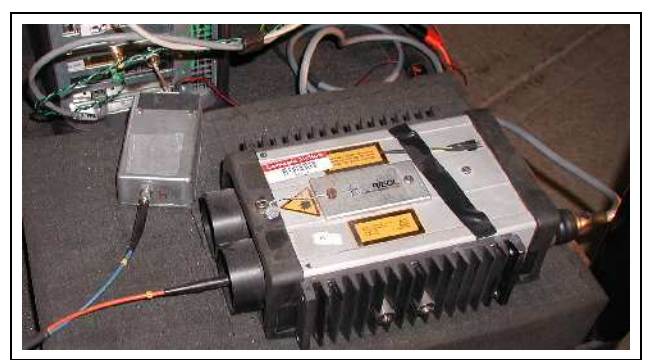

(c) Laser head and converter

Fig. 1. Sensor

The closest related work can be found in [16]. Schilling et al. present a laser radar to detect targets hidden by camouflage nets by extracting multi-echoes of a laser pulse waveform.

In opposition to the above mentioned lasers, we present a new compact and versatile sensor conceived with off-theshelf components, with one exception, the receiving circuitry. This laser provides a new sensing modality for mobile robots navigation specifically in outdoor environments.

\section{B. Laser waveform analysis}

Laser pulse waveform analysis has already been used to extract meaningful information from the illuminated scene. Various models of interaction of the laser beam with the environment has been proposed [17]. For example, models of light propagation into tree canopy have been proposed in order to inverse the observation model to extract bio-parameters like the leaf area index. Models have been also proposed to extract ice surface parameters (slope and terrain roughness, albedo) in polar regions from data collected by space-born laser. But such lasers are powerful, have large footprints (up to $65 \mathrm{~m}$ for SLICER) and emit long pulses (4 ns) at low repetition rate (40 Hz).

With our sensor and the applications we envisioned, we are in a complete different situation. Mobile robots need more versatile sensing, easy to use and deployable on small payload vehicles. We use a low power, short pulse, high repetition rate, and narrow beam laser. This type of laser returns a multi-modal waveform but the integration effect of the laser pulse is much more difficult to capture.

\section{SENSOR DESIGN}

\section{A. Sensor components}

The sensing device is made of five components: a turret, a laser head, an optical head, a digitizer, and an optical/DC converter. The experimental set-up can be seen in Figure 1 with a close view of the the turret and the converter. A block diagram of the sensor can be found in Figure 2.

The turret consists of the first two joints of a Mitsubishi Heavy Industries PA-10 industrial robot arm and is used as a pan-tilt scanning device [18]. This high performance robot, $\frac{1}{100} \mathrm{deg}$ angular accuracy and $100 \mathrm{deg} / \mathrm{s}$ angular rotation, was naturally selected because it was used by Carnegie Mellon University for EyeVision at the 2001 Superbowl, and is in use in the virtualized reality room at CMU [19].

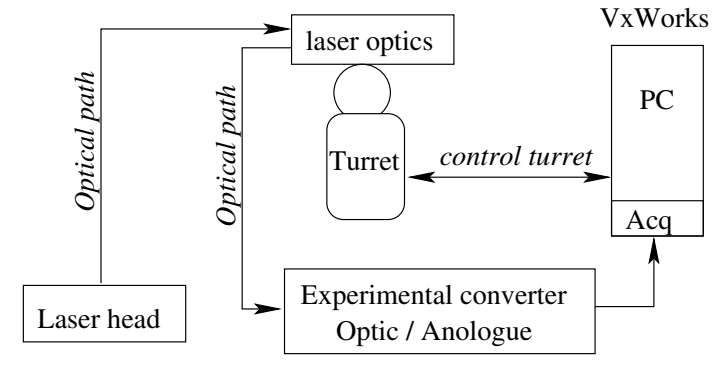

Fig. 2. Block diagram of the sensor components

The optical head is a Riegl MK36 and the range finder module is a Riegl LD90-3100 EHS-GF-HP, extremely highspeed. The measurement characteristics are: $\pm 10 \mathrm{~cm}$ accuracy, $10 \mathrm{~cm}$ resolution, $12000 \mathrm{~Hz}$ rate. The laser wavelength is in the near-infrared and the beam divergence is $3 \mathrm{mrad}$. We use an optical fiber to transmit the laser signal from the emitter of the laser head to the optical head and then to a receiver.

We do not use the laser receiver portion of the Riegl range finder, since it only provides a digital range output. Instead, we use a converter designed in our lab that provides a highbandwidth analog representation of the entire received laser return, as required for our study. This converter contains a fiber optic coupler, infrared filter, and PIN photo-diode to convert the received light into a tiny electrical current. A high-gain trans-impedance amplifier boosts this signal and conditions it appropriately to drive the coaxial cable leading into the digitizer. This amplifier was designed for another project, and unfortunately has a limited bandwidth of only $25 \mathrm{MHz}$.

The signal is then digitized using a 1Gs (1 ns) DP110 Acqiris Digitizer [20]. The card is installed in a PC running VxWorks that also controls the turret through an ARCNET connection. 1 Gs correspond to 1 sample per nanosecond or an optical path of $30 \mathrm{~cm}$ round trip or $15 \mathrm{~cm}$ in range. The acquisition card is configured to collect five waveforms consecutively and to average them. The peak location is determined by performing local polynomial interpolation.

To trigger the acquisition, we choose to have the laser head emitting continuously and to have a time reference target placed at a known distance in front of the optical head and rigidly attached to it. This time reference target partially occludes the laser beam and the resulting echo is used as a time reference. We use a $0.95 \mathrm{~cm}$ wide stick covered with an 
aluminum foil placed at $27 \mathrm{~cm}$ from the optical head. The laser beam at this distance is $3.8 \mathrm{~cm}$ in diameter. The size of the time reference target is set experimentally to produce a signal strong enough to achieve reliable triggering, while minimizing the amount of signal intercepted. Currently it occludes approximatively $\frac{1}{5}$ of the laser beam.

\section{B. Example}

Figure 3 shows an example of the signal captured by the sensor. Figure 3-(a) shows the scene used for this experiment: a set of four different wires strung horizontally in front of a distant wall. Figure 3-(b) shows the waveform of the returned signal produced by one laser pulse. The horizontal axis is time in nanosecond and the vertical is the amplitude in millivolt. The first mode is due to the time reference target, the second is due to the wire, and the last is due to the background wall. The difference between the position of the modes peak gave the duration of the light path.

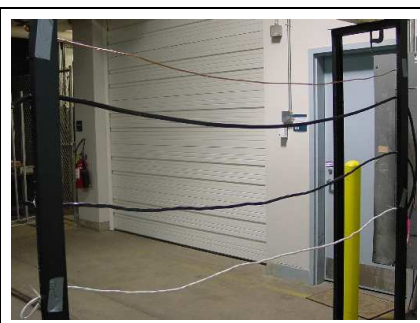

(a) Scene with wires

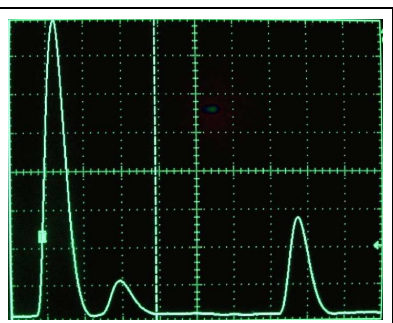

(b) Waveform
Fig. 3. Example of pulse waveform

\section{TOWARD LASER PULSE WAVEFORM ANALYSIS}

In this section, we take a closer look at the waveform of the signal produced by our sensor. It is important to determine if the mode shape is influenced by the target or if it is the result of sensor artifacts. We start with the shape of the mode. We then experiment with targets that we know are a common source of problems: planar discontinuities and porous surfaces.

\section{A. Local shape analysis}

Figure 4 shows different waveforms from the same target but seen at different distances. We used a white target, illuminated at near normal incidence. We observed that the intensity is not following the $\frac{1}{r^{2}}$ rule and we suspect the converter to be responsible for.

In addition we observed that the shape of the peak changes with the intensity of the returned signal. At short distances the peak has a long rise and a short trail. As the distance increases the peak becomes symmetric and then a heavy trail appears. Figure 5 shows the local shape of the peak for a white target at $11 \mathrm{~m}$ (short range) and $116 \mathrm{~m}$ (long range). The peak due to the time reference is not presented here.

This finding is expected to produce error in the range estimation. The kurtosis - the flatness of the curve - can be used to estimate the uncertainty of our range measurement. We use the kurtosis, in addition to the skewness, to measure

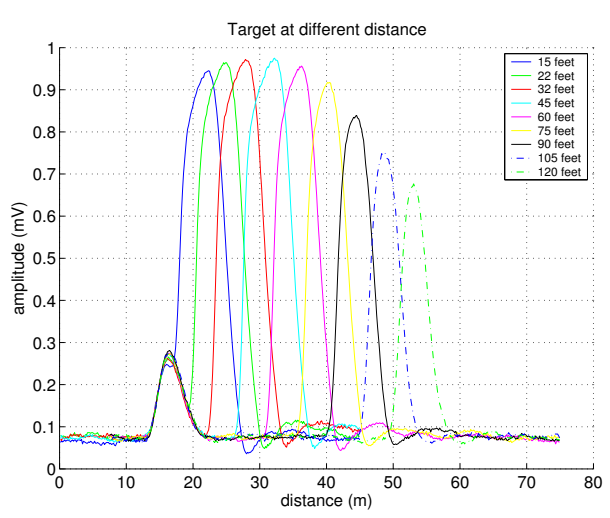

Fig. 4. Range influence

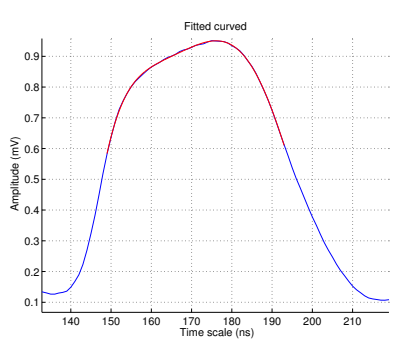

(a) $11 \mathrm{~m}$

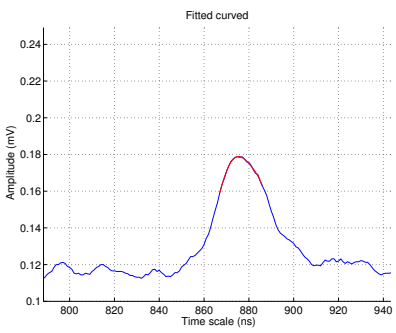

(b) $116 \mathrm{~m}$
Fig. 5. Local shape. In blue raw data, in red polynomial curve fitting

the shape deformation of the waveform in the case of a solid target seen at different incident angles. Results are presented in Table I. The incidence changes from normal incidence $\left(0^{\circ}\right)$ by 10 degrees increment.

TABLE I

WAVEFORM AND SOLID TARGET ORIENTATION

\begin{tabular}{c|ccc} 
Angle $^{o}$ & Peak position (ns) & Skewness & Kurtosis \\
\hline 0 & 411.770 & 5.278386 & 32.707100 \\
10 & 411.519 & 5.289305 & 32.917743 \\
20 & 411.791 & 5.098450 & 30.780768 \\
30 & 412.152 & 4.974963 & 29.516623 \\
40 & 411.500 & 4.522695 & 24.593181 \\
50 & 411.523 & 4.391199 & 22.986111 \\
60 & 412.693 & 4.050320 & 19.193124
\end{tabular}

As the incidence angle decreases, the kurtosis decreases and the skewness decreases. The peak becomes less sharp and more symmetric.

\section{B. Behavior at planar discontinuities}

We positioned a fixed and a mobile planar surface perpendicular to the viewing direction of the laser. The mobile surface can be moved incrementally in the vertical direction in order to occlude the background target. We collected waveforms for 11 different positions of the moving plate, they can be seen in Figure 6. One can observe the change of the amplitude of the two modes, as expected, and also the change of the shape of mode. Such phenomenon can be used to build a model of the sensor in order to estimate the surface area of the target illuminated. 


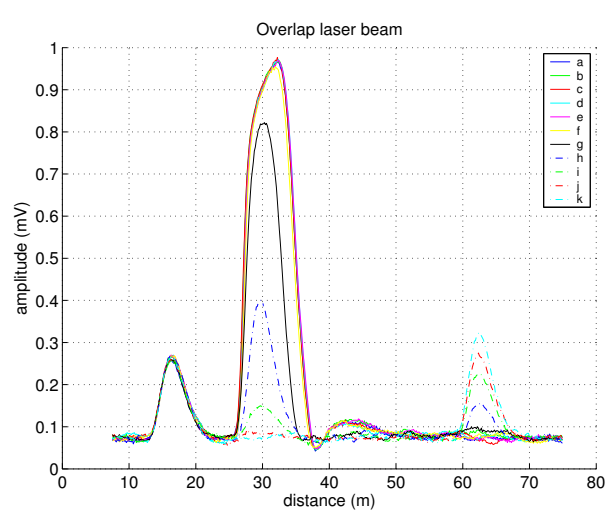

Fig. 6. Vertical discontinuity

\section{Mixed pixel detection}

Partial illumination of multiple targets with the same laser beam produces an error in range measurements called mixed pixels. As we presented earlier, methods exist to deal partially with such a problem indoors. Our sensing approach partial illuminations can be detected by looking at the raw data, the waveform, without making any assumption on the environment sensed. A fixed target was placed behind a mobile target at different distance $(1.75 \mathrm{~m}, 3.31 \mathrm{~m}, 4.85 \mathrm{~m}, 7.28 \mathrm{~m})$. The mobile target is used to occlude the fixed target. We go from far target complete illumination, to partial illumination of both targets, to close target full illumination. Results are presented in Figure 7. The existence of mixed pixel can be inferred, either by the presence of two overlapping Gaussian-like shapes with two peaks or by a single heavy tailed or rise single gaussian-like shape.

\section{Behavior with porous surface}

We compare laser reflection from a porous surface to a solid surface. The energy returned by a solid surface object comes locally, at the level of the laser footprint, from one or two surfaces in case of discontinuity. The energy returned by porous object will come from a multitude of small surfaces at potentially different depths. Porous surface objects include trees and vegetation, camouflage nets and wiry structures.

Figure 8 shows results obtained by illuminating a single layer and a double consecutive layer of a plastic net. The net is made of $5 \mathrm{~cm} \times 4.45 \mathrm{~cm}$ holes with $0.5 \mathrm{~cm}$ large plastic borders. We use the two layers, at the same depth, to produce a less permeable surface. In the Figure 8-(a) the single layer mesh is transparent. Figure 8-(b) shows several waveforms for the double layer mesh. Examples include partial and complete beam interception by the mesh.

\section{Standard EVALUation}

In this section we report on a set of standard tests performed with this laser: range, repeatability, range accuracy, range resolution, and color-range cross-talk. For all experiments described in the rest of the paper, we used targets made of mat Canson paper, unless otherwise noted. Measurements are performed at near normal incidence.

\section{A. Sensor range}

Figure 9 shows the waveform of the same target at different distance. The second peak occurs with the target at 5 meters. This is the minimum range measurement. Range measurements up to $116 \mathrm{~m}$ have been performed. The setting used to collect the ground truth limited the maximum range reachable.

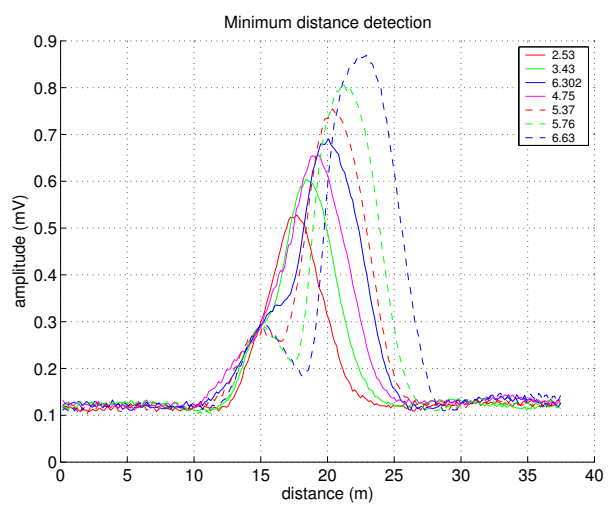

Fig. 9. Minimum range

\section{B. Color-Range cross-talk}

We illuminated at normal incidence colored targets. Waveforms recorded are reported in Figure 10. We observe a variation of the target peak location depending on the color of the target. The graph shows, in addition, a low, but above the noise, signal for a black target. However the flatness of the peak, in opposition to the sharp peak for the white target, does not allow a reliable range estimation. The shape of the red target signal cannot be explained at this point, but it was consistent over multiple tests.

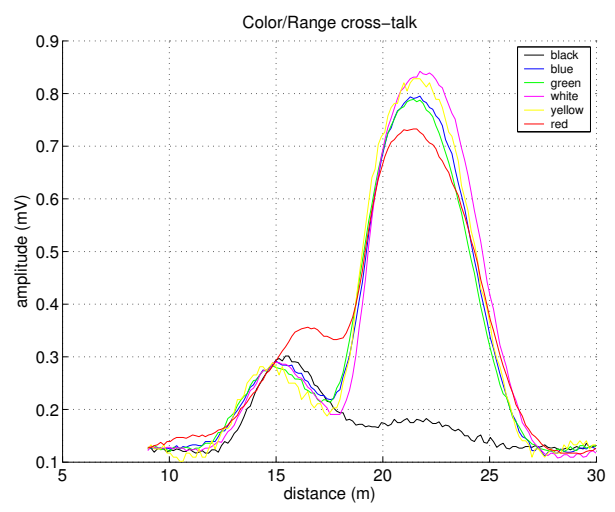

Fig. 10. Cross-talk

\section{Repeatability}

We are interested in the ability of the sensor to return consistent consecutive range measurements of the same target. A white target was illuminated at near normal incidence from a fixed distance. Figure 11 shows the envelop of the waveform defined as the maximum and minimum value for each sampled 


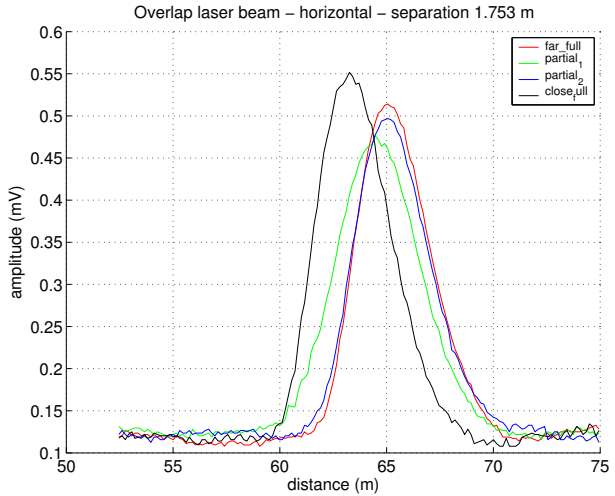

(a) $1.75 \mathrm{~m}$ separation

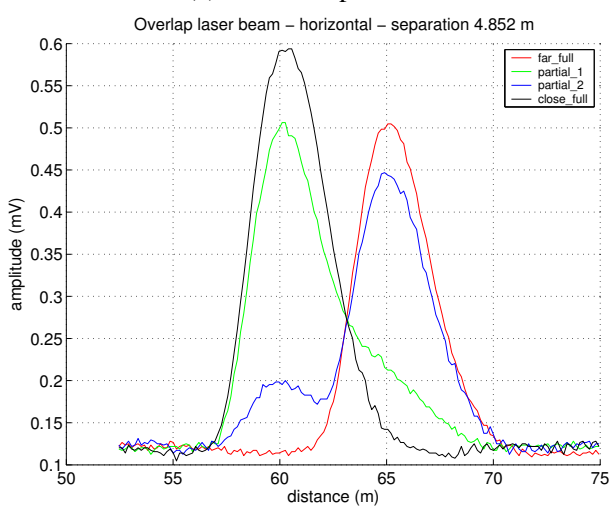

(c) $4.48 \mathrm{~m}$ separation

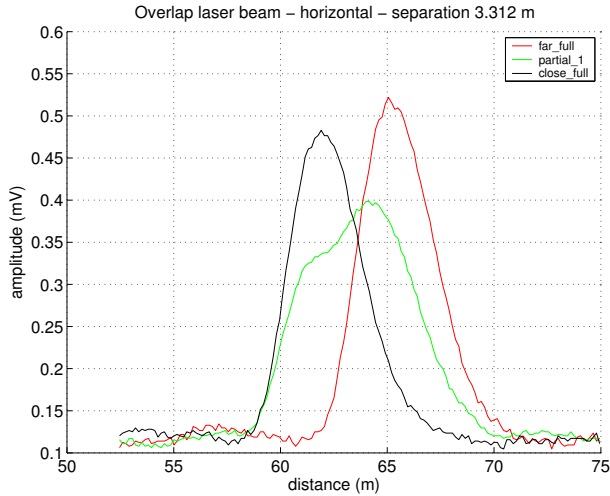

(b) $3.31 \mathrm{~m}$ separation

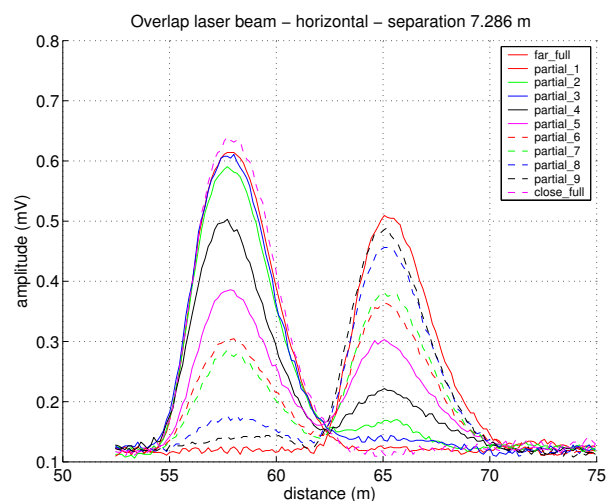

(d) $7.28 \mathrm{~m}$ separation

Fig. 7. Power of separation

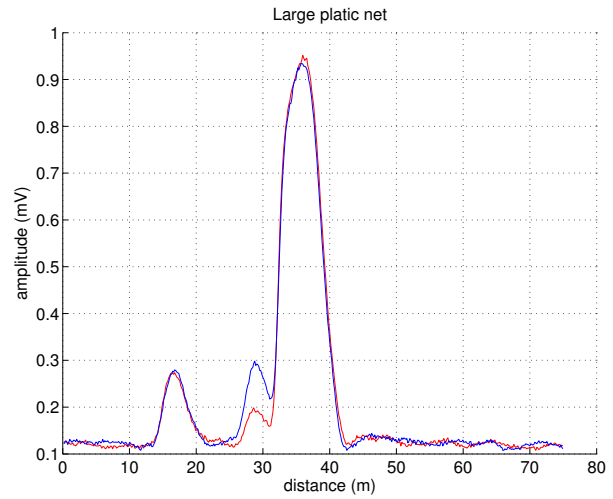

(a) Single net

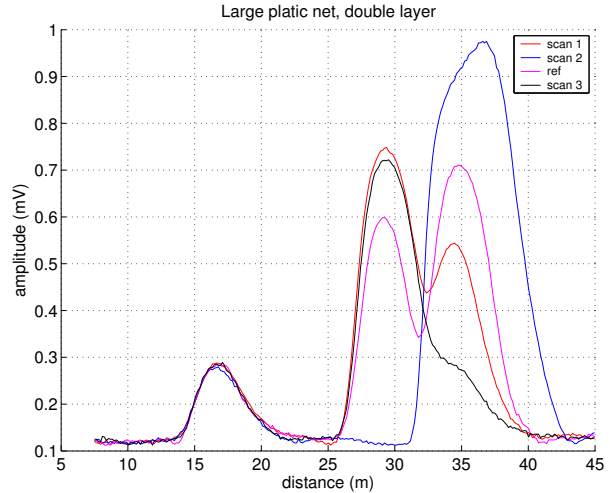

(b) Double net

Fig. 8. Porous surface illumination. Each graph contains several range measurement taken from different section of the mesh

measurement, over all waveform. The standard deviation of the position of the maximum is $0.4726 \mathrm{~ns}$ or $14.2 \mathrm{~cm}$.

\section{Range accuracy}

We set-up a white target on a mobile vertical platform, then tracked its position, our ground truth, with a theodolite and a prism mounted on the target. We maintained normal incidence with the laser. Figure 12 shows the range measurement versus the ground truth. At short range the laser behaves poorly, with large error due to the overlapping of the peaks produced by the reference distance stick and the target. Above 50 meters, we can achieve less than $7 \mathrm{~cm}$ accuracy with the local interpolation of the peak. In the worst case we got $20 \mathrm{~cm}$.

\section{E. Wiry and thin object detection}

We used a wire mesh net with $2.54 \mathrm{~cm}$ x $3.8 \mathrm{~cm}$ hexagonal pattern made of $0.9 \mathrm{~mm}$ diameter wire. We have been able to detect it up to $14.90 \mathrm{~m}$. We used a $1 \mathrm{~cm}$ diameter aluminum rod. We achieved detection up to $19.35 \mathrm{~m}$. The rod was aligned horizontally and the laser beam was centered on the target. 


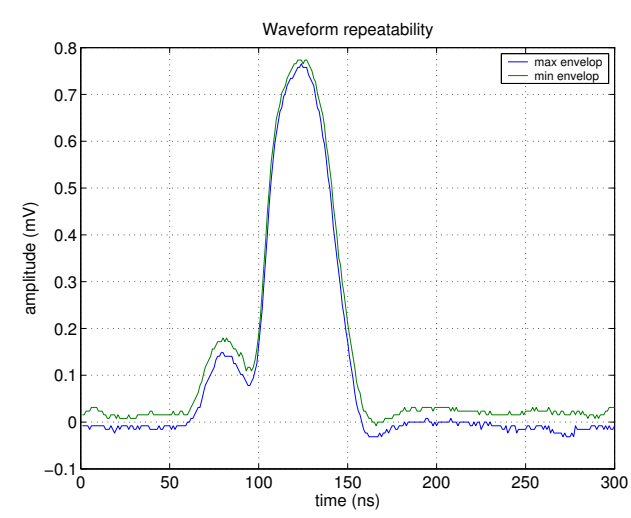

Fig. 11. Waveform repeatability

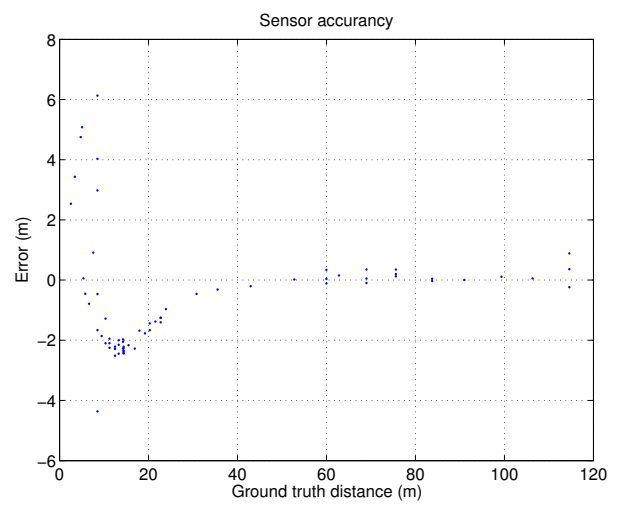

Fig. 12. Accuracy

\section{F. 3-D scene reconstruction}

Figure 13 shows an example of 3-D scene reconstruction. The scene is made of two wires with a flat panel and then a wall in the background. The laser was mounted on a cart. We scanned the scene using the pan-tilt head, detecting multiple echoes. The waveform averaging and peak extraction and local interpolation was performed on-line while scanning.

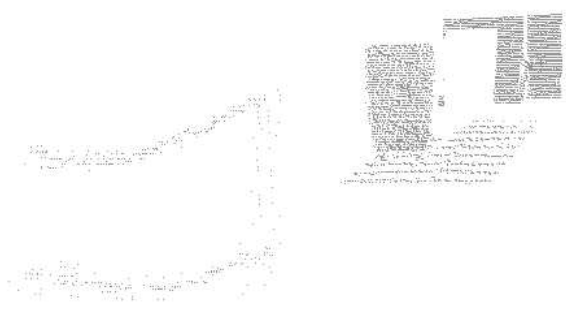

Fig. 13. 3D scenes reconstruction

\section{CONCLUSION}

In this paper we presented the design and evaluation of a new low-cost laser sensor that can record the full waveform of laser pulses bouncing back from illuminated targets. The main motivations for such a sensor is the detection of thin obstacles such as wires and targets hidden in vegetation for robotic applications. The use of off-the-shelf components helped us to build a low cost sensor offering a new sensing modality.

On one hand, we showed that the current system does not outperform the basic one echo per pulse range finder unit. We quantified its performances by evaluating standard characteristics. On the other hand, we showed that the analysis of the mode (distribution, shape) of the laser pulse waveform can produce new information (mixed pixel detection).

Our current research effort focuses on three axes: 1) improving the system design by acquiring a higher speed acquisition card, building a higher bandwidth converter and using a different time reference mechanism, 2) producing a more efficient time and space integration, 3) analyzing more thoroughly the waveform and its relationship to the target.

\section{REFERENCES}

[1] A. Foessel, "Scene modeling from motion-free radar sensing," Ph.D. dissertation, Robotics Institute, Carnegie Mellon University, Pittsburgh, PA, January 2002.

[2] J. Macedo, R. Manduchi, and L. Matthies, "Ladar-based discrimination of grass from obstacle for autonomous navigation," in Int. Symp. on Experimental Robotics, 2000.

[3] A. Castano and L. Matthies, "Foliage discimination using a rotating ladar," in IEEE Int. Conf. on Robotics and Automation, 2003.

[4] A. K. N. Vandapel, D. F. Huber and M. Hebert, "Natural terrain classification using 3-d ladar data," in IEEE Int. Conf. on Robotics and Automation, 2004.

[5] M. Hebert and N. Vandapel, "terrain classification techniques from ladar data for autonomous naviga tion," in Collaborative Technology Alliances Conference, May 2003.

[6] C. Ye and J. Borenstein, "Characterization of a 2d laser scanner for mobile robot ob stacle negotiation," in IEEE Int. Conf. on Robotics and Automation, 2002.

[7] M. Adams, "On-line gradient based surface discontinuity detection for outdoor scanning range sensors," in IEEE/RSJ Int. Conf. on Intelligent Robots and Systems, 2001.

[8] F. Tospann, M. Pirkl, and W. Gruener, "Multifunction 35-ghz fmcw radar with frequency scanning antenna for synthetic vision applications," in Proceedings of the SPIE, vol. 2463, 1995.

[9] K. Schulz, S. Scherbarth, and U. Fabry, "Hellas: obstacle warning system for helicopters," in Proceedings of the SPIE, vol. 4723, 2002.

[10] M. Nixon, "Improved passive obstacle detection using passive ranging," in Proceedings of the SPIE, vol. 4713, 2002.

[11] A. Ullrich, N. Studnicka, J. Riegl, and S. Orlandini, "Long-range highperformance time-of-flight-based 3d imaging sensors," in International Symposium on 3D Data Processing Visualization and Transmission, 2002.

[12] M. Shneier and al., "A repository of sensor data for autonomous driving research," in Proceedings of the SPIE Aerosense Conference, 2003.

[13] E. Baltsavias, "Airborne laser scanning: existing systems and firms and other resources," ISPR Journal of Photogrammetry \& Remote Sensing, vol. 54, 1999.

[14] H. A. et al., "Scannerless imaging pulsed-laser range finding," Journal of Optics A: Pure and Applied Optics, 2002.

[15] G. Iddan and G. Yahav, "3d imaging in the studio," in Three-Dimensional Image Capture and Applications IV, vol. SPIE Vol. 4298, 2001.

[16] B. Schilling, D. Barr, G. Templeton, L. Mizerka, and C. Trussel, "Threedimensional imaging of obscured targets by multiple-return laser radar," in Proceedings of the 2002 Army Science Conference, 2002.

[17] W. Ni-Meister, D. Jupp, and R. Dubayah, "Modeling lidar waveforms in heterogeneous and discrete canopies," IEEE Tran. on Geoscience and Remote Sensing, vol. 39, no. 9, 2001.

[18] K. Oonishi, N. Oonishi, and K. Shimoyama, "Producing and the latest development programs of the portable general purpose intelligent arm 'mitsubishi pa-10',' in Advanced Robotics, vol. 15, no. 3, 2001.

[19] B. Collins, O. Amidi, and T. Kanade, "An active camera system to acquiring multi-view video," in IEEE Int. Conf. on Image Processing, 2002.

[20] Acqiris Digitizers: User manual. 